\title{
Muscle atrophy and fatty infiltration after an acute rotator cuff repair in a sheep model
}

\author{
Tammy Luan 1 \\ Xuhui Liu1,2 \\ Jeremiah T. Easley ${ }^{3}$ \\ Bharat Ravishankar ${ }^{1,2}$ \\ Christian Puttlitz ${ }^{3}$ \\ Brian T. Feeley ${ }^{2}$ \\ 1 Department of Veterans Affairs, San Francisco Vet- \\ erans Affairs Medical Center, San Francisco, CA, \\ United States \\ 2 Department of Orthopaedic Surgery, University of \\ California, San Francisco, CA, United States \\ ${ }^{3}$ Colorado State University, Fort Collins, CO, USA
}

Corresponding author:

Brian T. Feeley

Department of Orthopaedic Surgery

University of California

1500 Owens Ave, Box 3004

94158 San Francisco, USA

E-mail: feeleyb@orthosurg.ucsf.edu

\section{Summary}

Introduction: rotator cuff tears (RCTs) are the most common tendon injury seen in orthopedic patients. Muscle atrophy and fatty infiltration of the muscle are crucial factors that dictate the outcome following rotator cuff surgery. Though less studied in humans, rotator cuff muscle fibrosis has been seen in animal models as well and may influence outcomes as well. The purpose of this study was to determine if the rotator cuff would develop muscle changes even in the setting of an acute repair in a sheep model. We hypothesized that fatty infiltration and fibrosis would be present even after an acute repair six months after initial surgery.

Methods: twelve female adult sheep underwent an acute rotator cuff tear and immediate repair on the right shoulder. The left shoulder served as a control and did not undergo a tear or a repair. Six months following acute rotator cuff repairs, sheep muscles were harvested to study atrophy, fatty infiltration, and fibrosis by histological analysis, western blotting, and reverse transcription polymerase chain reaction (RT-PCR).

Results: the repair group demonstrated an increase expression of muscle atrophy, fatty infil- tration, and fibrosis related genes. Significantly increased adipocytes, muscle fatty infiltration, and collagen deposition was observed in rotator cuff muscles in the tendon repair group compared to the control group.

Conclusions: rotator cuff muscle undergoes degradation changes including fatty infiltration and fibrosis even after the tendons are repair immediately after rupture.

Level of Evidence: Basic Science Study.

KEY WORDS: acute rotator cuff repair, fatty infiltration, fibrosis, SREBP-1, PPAR $\gamma$.

\section{Introduction}

Rotator cuff tears (RCTs) are the most common shoulder injury seen by orthopedic surgeons and are especially frequent among the aging patient population. The prevalence of full thickness RCTs is estimated between 15 and $51 \%$, with higher rates in the elderly population ${ }^{1}$. Although repair of small tears is successful in alleviating pain and improving shoulder biomechanics, successful repair of larger RCTs still remains a challenge $\mathrm{e}^{2,3}$. As the incidence of rotator cuff injuries continues to rise with an aging patient population, management and knowledge of the etiopathogenesis of RCTs ${ }^{4}$ is a topic of concern.

Several muscle-based factors have been identified to affect the outcome of rotator cuff repairs. It has been demonstrated that the degree of atrophy correlates with the size of the tear and, more importantly, with clinical outcomes ${ }^{5,6}$. Similarly, fatty infiltration has been shown to also correlate with poor outcomes following rotator cuff repair ${ }^{6,7}$. Although the clinical effects of muscle fibrosis on rotator cuff injuries are not well quantified, muscle fibrosis is an important contributor to muscle stiffness and is seen in many other muscle injury models ${ }^{8}$. Thus, these three physiological traits, muscle atrophy, fatty infiltration, and muscle fibrosis, are likely key factors governing outcome after rotator cuff injury and repair.

Animal models are useful tools to study the molecular mechanisms of muscle atrophy, fatty infiltration, and fibrosis that are observed in the setting of rotator cuff tears in patients. While there are multiple RCTs studies conducted in rat or mouse models ${ }^{9-12}$, the sheep model, as a larger animal model, may more closely mirror the pathophysiologic changes observed in human $R C T s^{13}$ since the infraspinatus tendons of sheep 
are similar to those of human rotator cuff in size and shape ${ }^{14}$. Although the development of muscle atrophy and fatty infiltration are correlated with chronic $\mathrm{RCTs}{ }^{15-17}$, it is unclear if acute rotator cuff injuries result in any muscle injury, if at all.

Thus, the purpose of this study was to evaluate how a sheep model of acute repair responds following recovery. Specifically, we wished to determine if fatty infiltration and fibrosis developed in an acute RCT after immediate repair. We hypothesized that fatty infiltration and fibrosis would be present even after an acute repair six months after initial surgery.

\section{Methods}

Our study meets the ethical standards of the journal ${ }^{18}$.

\section{Surgical procedure}

Surgeries were performed on twelve female adult sheep that were supplied by Colorado State University's Surgical Research Laboratory (Fort Collins, Colorado, CO). All sheep underwent an acute rotator cuff tear and immediate repair on the right shoulders while the left shoulders remained untreated as the controls. The facility's Institutional and Use Committee (IACUC) approved all procedures and handling of the animals.

Each ewe was fasted and premedicated with $1 \mathrm{gram}$ of oral phenylbutazone (Bute Boluses, VEDCO, Inc., St. Joseph, MO, USA), procaine G penicillin $(22,000$ units/kg, SQ - PenOne Pro ${ }^{\mathrm{TM}}$, Norbrook Laboratories Limited, Newry, Ireland), and two transdermal fentanyl patches (100 mcg, $50 \mathrm{mcg}$ - Fentanyl Transdemal System, Watson Pharma, Inc., Corona, CA, USA) 24 hours prior to surgery. The auricular vein and artery were catheterized and anesthesia was induced using a combination of ketamine $\mathrm{HCl}(3.3 \mathrm{mg} / \mathrm{kg} \mathrm{IV} \mathrm{-}$ Ketaset $^{\mathrm{TM}}$ Boerhinger-Ingelheim, St. Joseph, MO, USA) and diazepam (0.1 mg/kg IV - Diazepam, Hospira, Inc., Lake Forest, IL, USA). Following anesthetic induction, the sheep were intubated with a cuffed endotracheal tube, placed in dorsal recumbency and maintained on isoflurane $\left(1.5 \%-3 \%\right.$ - Fluriso ${ }^{\mathrm{TM}}$, Norbrook Laboratories Limited, Newry, Ireland) with $100 \%$ oxygen using positive pressure ventilation (20 $\mathrm{cm} \mathrm{H}_{2} \mathrm{O}$ ) for the duration of the procedure.

The sheep were placed under general anesthesia in left lateral recumbency. Using sterile conditions, the skin over the right shoulder joint was prepared for aseptic surgery using alternating scrubs of povidone-iodine (Betadine) and alcohol. The shoulder joint was draped for aseptic surgery. A $12 \mathrm{~cm}$ skin incision was made over the point of the shoulder joint. The m. subcutaneous coli was divided in line with the incision. The $\mathrm{m}$. deltoideus was split along the tendinous division between its acromial and scapular heads. The superficial head and insertion of infraspinatus tendon was isolated. The insertion of the infraspinatus tendon to the greater tuberosity of the humerus was sharply transected, and any remaining fibrocartilage at the insertion was removed. Following detachment of the infraspinatus tendon, a hall air drill and burr were used to create a bed of bleeding bone at the footprint of the infraspinatous tendon. Two pilot holes were drilled into the footprint approximately $1 \mathrm{~cm}$ apart and the holes were tapped to accommodate the sutures anchors. Two $5.5 \mathrm{~mm}$ suture anchors were deployed into the dense bone of the medial footprint of the proximal humerus (one anchor cranially and one anchor caudally). The suture anchors were placed approximately $1 \mathrm{~cm}$ apart and $2 \mathrm{~mm}$ to $4 \mathrm{~mm}$ deep to the cortex. Sutures were passed from inferior to superior through the infraspinatus tendon and tied in simple mattress fashion on the superior surface of the tendon. After suturing the tendon back to the footprint, the surgical site was lavaged with sterile saline. The acromial head of the $\mathrm{m}$. deltoid us was returned to its more cranial position and attached to the brachial fascia using a simple continuous suture pattern using size 2/0 absorbable suture material (Polysorb). The brachial fascia and subcutaneous tissues were closed as separate layers using $2 / 0$ Polysorb while stainless steel staples (Proximate; Ethicon) were used for the skin closure. Six months later, sheep were humanely euthanized by intravenous barbiturate overdose (Pentobarbitone sodium, $88 \mathrm{mg} / \mathrm{kg}$ ) according to the guidelines set forth by the American Veterinary Medical Association in 2013. Based on our previous rat model studies $9,11,12,19$, at least four animals are needed to determine a significant difference in protein expression levels with the following power assumptions $a=0.05, \beta=0.80$. In order to improve the power of our result, we used six sheep per analysis group (12 sheep total).

\section{Muscle harvest}

Sheep were sacrificed six months after surgery. Infraspinatus muscles were harvested and the remaining tendon and scar tissue was removed at the muscle/ tendon junction. Multiple samples were taken from the muscle region of the infraspinatus as close to the tendon as possible. Twelve muscles from six randomly selected sheep were used for histology analysis and eighteen samples from the remaining six sheep were divided in half for either protein or RNA extraction ( $\mathrm{N}=6$ in each group). The tissue for biochemical analysis was subsequently homogenized in T-PER solution (Pierce Biotechnology Inc. Rockford, IL.) with a protease inhibitor cocktail (Sigma-Aldrich Inc. St Louis, MO) for total protein extraction or Trizol ${ }^{\circledR}$ solution (Invitrogen Inc. Carlsbad, CA) for total RNA extraction.

\section{Total collagen staining}

Muscle samples for histology analysis were fixed in 4\% paraformaldehyde. After fixation, the tissues were processed and dehydrated following cycle: $70 \%$ 
ethanol 2 times, 1 hour each; 80\% ethanol, 2 times, 1 hour each; 95\% ethanol, 2 times, 1 hour each; 100\% ethanol, 3 times, 1 hour each; xylene, 3 times, 1 hour each; and paraffin wax, 2 times, 1.5 hour each. Following tissue processing, muscles were embedded in paraffin and sectioned at a thickness of $5 \mu \mathrm{m}$. Masson's Trichrome Staining (Fisher Scientific Inc., Waltham, $\mathrm{MA}$ ) was performed to examine differences in total extracellular matrix collagen between control and repair group muscles following six months.

\section{Western-blot analysis}

After determining the protein concentration from the homogenized samples, fifty micrograms of protein was loaded on $10 \%$ NUPAGE Bis-Tris gels and transferred to Trans-Blot Turbo mini PVDF membranes (Biorad) that were blocked and incubated in primary and secondary antibodies. The following rabbit-anti-sheep primary antibodies were used at dilutions of 1:200: SREBP-120, 21 (Novus Biologicals, Lit-

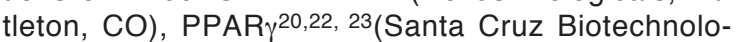
gy, Santa Cruz, CA), p-AKT ${ }^{11,24}$ (Cell Signaling Technology), and p-mTOR ${ }^{11,24}$ (Cell Signaling Technology). IRDye 800 CW Goat anti-Rabbit IgG (H+L) secondary antibody (LI-COR Biosciences, Lincoln, NE) was used at a dilution of 1:10,000. Blots were imaged using the Odyssey Infrared Imaging System (LI-COR Biosciences).

\section{Quantitative reverse transcription polymerase} chain reaction ( $q R T-P C R$ )

Isolated RNA was quantified and normalized $(1 \mu \mathrm{g})$ to synthesize cDNA using a Transcriptor First Strand cDNA Synthesis Kit (Roche Applied Bioscience Inc., Indianapolis, IN.). qRT-PCR was performed to quantify the expression of aSMA ${ }^{25,26}, \mathrm{Col}-1^{26}, \mathrm{Col}-3^{26}$ MuRF-111, 27, MAFbx ${ }^{11,27}, \mathrm{MMP}^{13}{ }^{28}, 29, \mathrm{PPAR} \gamma$, and
SREBP-1 using a Light Cycler 480 SYBR Green I Master kit (Roche Applied Bioscience Inc.). Primer sequences are listed in Table 1. Amplification reactions were performed with 40 cycles of $\left(95^{\circ} \mathrm{C}\right.$ for 10 $\mathrm{min} ; 58^{\circ} \mathrm{C}$ for $30 \mathrm{~s}$; and $72^{\circ} \mathrm{C}$ for $1 \mathrm{~min}$ ), and normalized to b-actin. Fold change in mRNA expression was calculated by using $\triangle \triangle \mathrm{CT}$.

\section{Results}

All animals tolerated the surgery well and survived with no complications. The forelimbs regained function following rotator cuff repairs and histologic analyses showed healing, with a certain amount of scar tissue, of rotator cuff tendons after repair (Fig. 1).

\section{Significant increase in muscle fibrosis after repair}

Muscle fibrosis was assessed histologically with Masson's Trichrome stain. The repair group showed a modest increase in fibrosis compared to the control group as indicated by the greater amount of fibroblast-like cells infiltrated into the space between myofibers in the muscle (Fig. 2). Fibrotic tissue was uniformly distributed throughout the muscle samples. Real-time RT-PCR of Col-1, Col-3, aSMA, and MMP13 supported our histology results with increased mRNA expression of these fibrotic makers in the repaired samples compared to the control samples (Fig. 4). All three genes demonstrated greater than a 4 -fold increase in mRNA expression in the surgical side compared to the control side.

\section{Repair samples have greater fatty infiltration com- pared to control samples}

Histology demonstrated increased adipocytes in the repair group compared to the untreated control group

Table 1. Primers used for qRT-PCR. Beta-actin (b-Actin); collagen, type 1, alpha 1 (Col I); collagen type III (Col III); alpha smooth muscle actin (aSMA); muscle RING finger-1 (MuRF-1); F-box only protein 32 (MAFbx); matric metallopeptidase 13 (MMP-13); peroxisome proliferator-activated receptor gamma (PPAR $\gamma$ ); and sterol regulatory element-binding protein 1 (SREBP-1).

\begin{tabular}{|c|c|c|}
\hline Gene & Forward $\left(5^{\prime} \rightarrow 3^{\prime}\right)$ & Reverse $\left(5^{\prime} \rightarrow 3^{\prime}\right)$ \\
\hline$b$-Actin & CGTGGCCATCCAGGCTGTGCTGTCC & CGTGGCCATCCAGGCTGTGCTGTCC \\
\hline Col $1 a$ & TACCATGACCGAGACGTGTG & GGACTTTGGCGTTAGGACAG \\
\hline Col IIla & CTGCCATTGCTGGTGTTG & TACGGGAACCATCAGGACTA \\
\hline aSMA & GTGTGTGACAATGGCTCTGG & TCTCAAAGTCCAGGGCTACG \\
\hline MAFbx & CTACGTCGTACGGCCTGC & CTCGGAGAAGTGGTACTGGC \\
\hline$M u R F-1$ & TGTGCCAACGACATCTTCCA & GATGATGTTCTCCACCAGCA \\
\hline$P P A R \gamma$ & CTTGCTGTGGGGATGTCTC & GGTCAGCAGACTCTGGGTTC \\
\hline SREBP-1 & CTGCTATGCAGGCAGCAC & GGTTGATGGGCAGCTTGT \\
\hline$M M P-13$ & GCCAGAACTTCCCAACCGTA & GTGAAGGGCTGCACTGATCT \\
\hline
\end{tabular}


(Fig. 2). Western blot analysis of the rotator cuff muscles indicated a significant increase in the expression of pro-adipogeneic PPAR $\gamma$ and SREBP-1 proteins in the repair group compared to the untreated group (Fig. 3). Real-time RT-PCR confirmed the Western blot data by demonstrating that the expression of SREBP-1 and PPAR $\gamma$ were significantly increased in the muscles of sheep six months after surgery (Fig. 4). There was a 6-fold increase in PPAR $\gamma$, and a 4fold increase in SREBP-1 expression.

\section{Up-regulation of atrophy related expression fol- lowing repair}

Real-time RT-PCR revealed expression of atrophy genes, MuRF-1 and MAFbx, was increased in the repaired group compared to the control group (Fig. 4).

\section{Discussion}

The primary goal of this investigation was to determine if an acute rotator cuff repair in a sheep model would result in pathophysiologic changes within the muscle. It has been shown in a rat model that repairing either acutely or chronically torn rotator cuff tears cause damage to the muscle fibers and that the degree of injury is more severe in the chronically torn rotator cuff muscles ${ }^{30}$. Thus, we hypothesized that fatty infiltration and fibrosis would be present in a sheep model even after an acute repair six months following

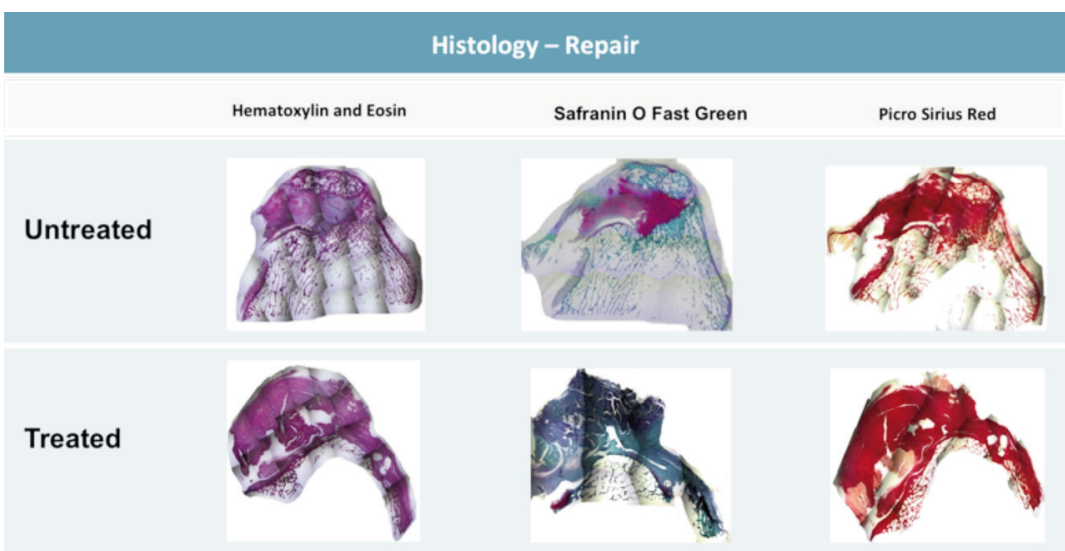

Histology - Trichrome Staining (10X Lens)
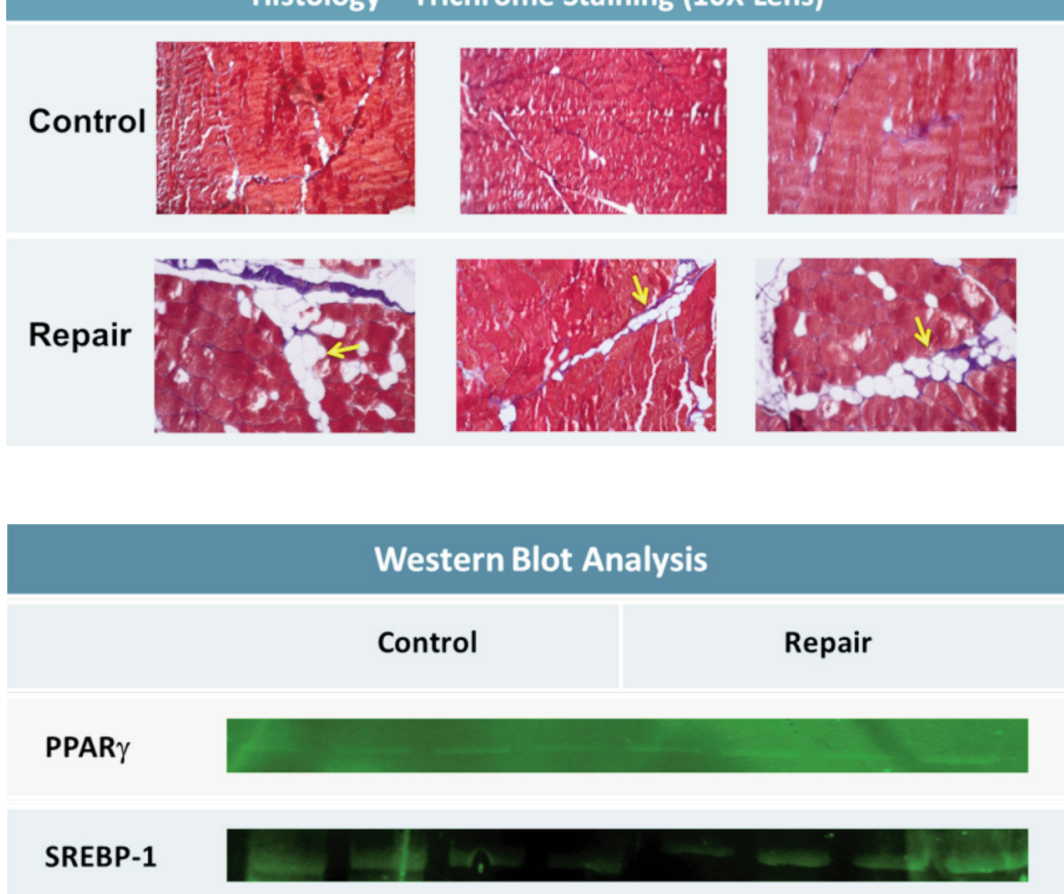

Figure 1. Hemotoxylin and Eosin, Safarin O Fast Green, and Picro Siruis Red histology analyses indicate all animals healed and regained function following acute repairs.

Figure 2. Typical Masson's Trichrome staining of sheep muscles from control and repair sides 6 months following tear and immediate repair. A significantly greater amount of collagen was deposited between myofibers in the repair group muscle. The repair group displayed increased adipocytes in comparison to the control group.

Figure 3. Western-blot showed a significant increase in PPAR $\gamma$ and SREBP-1 protein expression in the repair group compared to the control group. 


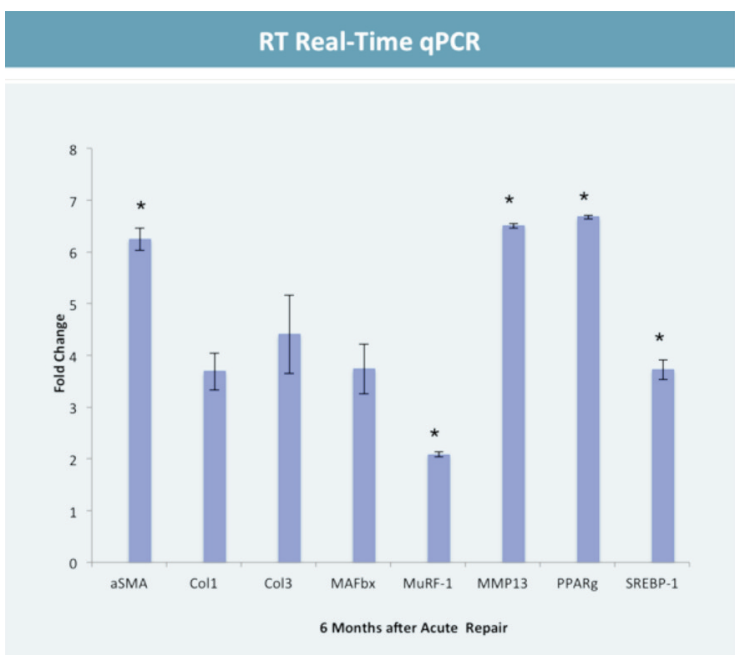

Figure 4. Real-time RT-PCR showed a significant increase in PPAR $\gamma$, SREBP-1, MuRF-1, MAFbx, aSMA, Col-1, Col3 , and MMP-13 gene expression in the repair side compared to the control side. $\left(\mathrm{N}=6,{ }^{*}\right.$ indicates $\left.\mathrm{P}<0.05\right)$.

initial surgery. Our results reveal muscle changes develop in an acute rotator cuff injury and repair. Specifically, our study demonstrates an increase in fibrosis and fatty infiltration following acute repair of a RCT. In addition, our data indicate we were able to track muscle changes both histologically and via altered gene expression six months after an acute repair, suggesting the rotator cuff muscle is susceptible to long term changes after an induced injury.

Akt and mTOR are critical proteins in the muscle mass homeostasis pathway that regulate muscle size and development of atrophy ${ }^{31}$. Alterations in Akt/mTOR activity result in changes in gene expression of the pro-atrophy related genes including MurF1 and MAFbx ${ }^{11}, 27$. These genes regulate atrophy through increased ubiquitin-proteosome protein breakdown. Akt and mTOR are also linked to other important pathways including the SREBP-1/adipogenesis pathway ${ }^{32}$ and TGF-B and pro-fibrotic pathways ${ }^{33}, 34$. Previous studies in small animal model show that Akt and mTOR have altered gene expression patterns after mechanical unloading and denervation, and respond differently to these signals ${ }^{11}$. There are conflicting data on the progression of atrophy following rotator cuff repairs: some studies report muscle atrophy worsens and does not improve after successful repair ${ }^{35,36}$, while other studies report muscle atrophy improves after successful cuff repair ${ }^{37-39}$. In our acute repair sheep model, we observed significant increases in atrophy-related gene expression of MuRF-1 and MAFbx. At a single, specific time point, it is difficult to determine the significance of Akt/mTOR protein expression changes ${ }^{40}$. Likewise, we were unable to determine differences in Akt/mTOR activity that is based on its phosphorylation state in the sheep model since there are no sheep-specific antibodies and non sheep-specific antibodies were inadequate for western blot analysis. However, the persistent in- crease in atrophy related gene expression in MuRF-1 and MAFbx suggests that muscle can be at risk for developing atrophy or increased protein turnover even after an acute repair. Clinically, this suggests that an immediate repair following an acute RCT may not entirely eliminate the risk for muscle atrophy development.

Fatty infiltration has been correlated with poor functional outcomes following RCT repairs ${ }^{16,} 41$. However, whether fatty infiltration improves, worsens, or halts after surgical repair is uncertain. Some studies show that fatty infiltration worsens and is irreversible following successful repairs ${ }^{3,42,36}$, yet other studies demonstrate that fatty infiltration improves following repairs $^{37,38}$. Previous studies have shown that pro-adipogenic factors including PPAR $\gamma$ and SREBP-1 are elevated in both large and small animal models of rotator cuff injury. In a clinical study, there was also altered adipogenic gene expression in different size cuff tears, suggesting that the expression of these genes is more complicated than once thought ${ }^{43}$. In this study, there was a significant increase of fat-related transcription factors, SREBP-1 and PPAR $\gamma$, in our acute repair model at the protein and mRNA expression level, as well as a moderate amount of fat present in histologic samples. These data suggest not only that fatty infiltration can develop in the rotator cuff muscles under acute conditions, but also that immediate repairs following RCTs do not eliminate the risk of rotator cuff fatty infiltration from occurring. Future studies can examine the biomechanical properties of these tissues to see how this small amount of fat within the muscle can result in altered mechanical outcomes of the muscle.

While muscle atrophy and fatty infiltration are well-described factors of successive rotator cuff repairs, muscle fibrosis is also thought to be an influential factor dictating the outcomes of large and massive $\mathrm{RCTs}^{8}$. Unlike muscle atrophy and fatty infiltration, muscle fibrosis is difficult to quantify in a clinical setting as it is not routinely evaluated by clinical MRI or ultrasound imaging. In a laboratory setting, the mechanical consequences of muscle fibrosis are well defined. Increased fibrosis results in decreased muscle compliance and increased muscle stiffness ${ }^{8}$. As a result of decreased muscle and tendon compliance, presumably due to muscle fibrosis, shoulder surgeons often require advanced techniques to advance stiff, noncompliant muscle to reduce the chronic tear back to greater tuberosity 44,45 . However, even these advanced techniques do not produce favorable results. Kim et al. ${ }^{46}$, reviewing the results of interval slide techniques to advance stiff, retracted RCTs, found a posterior interval slide to release the rotator cuff resulted in $91 \%$ retear rate at 2-year follow-up. Tissue engineering of collagen-based extracellular matrices can possibly serve as augmentation to the surgical re-attachment of large tears of rotator cuff tendons as Schlegel et al. engineered a highly porous, low modulus reconstituted collagen capable of inducing formation of new tendon-like tissue when sutured to the superficial surface of the infraspinatus 
tendons of adult sheep ${ }^{47}$. In the current study, our data show an increase in fibrosis in the acute repair model. There is a consistent upregulation of multiple profibrotic genes at 6 months. This suggests muscle fibrosis can occur in the rotator cuff in an acute repair setting. Also, our results indicate immediate repairs following acute RCTs do not significantly decrease the likelihood of developing fibrosis.

Overall these data suggest an acute rotator cuff injury results in similar muscle changes that are correlated with a chronic rotator cuff injury. Furthermore, these data have important clinical implications because even six months following an immediate repair of a RCT there are still structural and molecular alterations that promote the development of fatty infiltration and fibrosis in the rotator cuff muscles. In addition, this study suggest that this model may be clinically relevant for the development of potential therapeutic modalities to decrease fatty infiltration and fibrosis following RCTs as it appears from this study repair alone is not sufficient.

Although our study revealed fatty infiltration and fibrosis develop even in the acute setting of a tear and repair, there are several limitations to this study. Like other animal studies, the anatomy and mechanics of a sheep shoulder are different from that of a human shoulder. Therefore, although the sheep model shows similar changes to what is seen clinically, the results from this study may not be directly translated into clinical practice. Second, the amount of fibrosis and fatty infiltration were not quantified in the histological sections or biochemical assay, but rather we used a qualitative review. Future studies will quantitatively evaluate fibrosis and fatty infiltration changes. Third, although we examined atrophy related gene expression, we did not record sheep muscle weights and therefore could not compare muscle weights between repair and control side. Wet weights of muscle will be included in future studies. The evaluation of protein expression was also limited due to the limited number of sheep-specific antibodies for western blot and immune histochemistry analysis. Compared to small animal models, there are relatively few proteins that can be easily studied with conventional protein assays in a sheep model. In this study we were unable to determine Akt/mTOR activation with phosphorylation states with western blot analysis, which limits the conclusions that can be made in this aspect of the study. Future work will elucidate the protein expression of phosphorlyated states of Akt/mTOR following acute repair of a RCT. Similarly, RT-PCR and gene expression is limited by the lack of well-defined primers for key genes in sheep studies. We developed specific primers based on previously published studies and published gene sequence, but a more comprehensive panel of gene expression is limited at this time.

\section{Conclusions}

In summary, the rotator cuff develops muscle changes even in the setting of an acute repair in a sheep mod- el. Furthermore, fatty infiltration and fibrosis are significantly increased after an acute rotator cuff. These results suggest the clinical importance of developing potential therapeutic modalities to reduce fatty infiltration and fibrosis; as evidently, an immediate repair following a rotator cuff tear is not sufficient.

\section{Conflicts of Interest and Source of Funding}

No Authors have any conflicts of interest to disclose. Funding was provided from a grant from the OREF (Young Investigator Grant).

\section{Study Approval}

All animal procedures were approved by our IACUC (A3476-01).

\section{References}

1. Chaudhury S, Dines JS, Delos D, Warren RF, Voigt C, Rodeo SA. Role of fatty infiltration in the pathophysiology and outcomes of rotator cuff tears. Arthritis Care \& Res. 2012;64(1): 76-82.

2. Cofield RH, Parvizi J, Hoffmeyer PJ, Lanzer WL, Ilstrup DM, Rowland CM. Surgical repair of chronic rotator cuff tears: a prospective long-term study. J Bone Joint Surg Am. 2001;83$A(1): 71-71$.

3. Gerber C, Meyer DC, Schneeberger A, Hoppeler H, von Rechenberg B. Effect of tendon release and delayed repair on the structure of the muscles of the rotator cuff: an experiment study in sheep. J Bone Joint Surg Am. 2004;86-A(9):19731982.

4. Gia Via A, De Cupis M, Spoliti M, Oliva F. Clinical and biological aspects of rotator cuff tears. Muscles, Ligaments and Tendons Journal. 2013;3(2):70-79.

5. Gerber C, Meyer DC, Frey E, et al. Neer Award 2007: Reversion of structural muscle changes caused by chronic rotator cuff tears using continuous musculotendinous traction. An experimental study in sheep. J Shoulder Elbow Surg. 2009; 18(2):163-171.

6. Gladstone JN, Bishop JY, Lo IK, Flatow EL. Fatty infiltration and atrophy of the rotator cuff do not improve after rotator cuff repair and correlate with poor functional outcome. Am J Sports Med. 2007;35(5):719-728.

7. Pop M, Ghugre NR, Ramanan V, et al. Quantification of fibrosis in infarcted swine hearts by ex vivo late gadolinium-enhancement and diffusion-weighted MRI methods. Phys Med Biol. 2013;58(5):5009-5028.

8. Lieber RL, Ward SR. Cellular mechanisms of tissue fibrosis. 4. Structural and functional consequences of skeletal muscle fibrosis. Am J Physiol Cell Physiol. 2013;305(3):241-252.

9. Joshi SK, Liu X, Samagh SP, et al. mTOR regulates fatty infiltration through SREBP-1 and PPARg after a combined massive rotator cuff tear and suprascapular nerve injury in rats. $J$ Orthop Res. 2013;31(5):724-730.

10. Laron D, Samagh SP, Liu X, Feeley BT. Muscle degeneration in rotator cuff tears. J Shoulder Elbow Surg. 2012;21(2):164-174.

11. Liu X, Joshi SK, Samagh SP, et al. Evaluation of Akt/mTOR activity in muscle atrophy after rotator cuff tears in a rat model. J Orthop Res. 2012;30(9):1440-1446.

12. Liu X, Manzano G, Kim HT, Feeley BT. A rat model of massive rotator cuff tears. J Orthop Res. 2011;29(4):588-595. 
13. Turner AS. Experiences with sheep as an animal model for shoulder surgery: strengths and shortcomings. J Shoulder Elbow Surg. 2007;16:158-163.

14. Gerber C, Schneeberger AG, Beck M, Schiegel U. Mechanical strength of repairs of the rotator cuff. J Bone Joint Surg.1994; 76(3):371-380.

15. Fehringer EV, Sun J, Van Oeveren LS, Keller BK, Matsen FA 3rd. Full thickness rotator cuff tear prevalence and correlation with function and co-morbidities in patients sixty-five years and older. J Shoulder Elbow Surg. 2008;17(6):881-885.

16. Yamaguchi K, Tetro AM, Blam O, EvanoffBA, Teefey SA, Middleton WD. Natural history of asymptomatic rotator cuff tears: a longitudinal analysis of asymptomatic tears detected sonographically. J Shoulder Elbow Surg. 2001;10(3):199-203.

17. Beeler S, EkET, Gerber C. A Comparative Analysis of Fatty Infiltration and Muscle Atrophy in Patients with Chronic Rotator Cuff Tears and Suprascapular Neuropathy. J Shoulder Elbow Surg. 2013;11:1537-1546.

18. Padulo J, Oliva F, Frizziero A, Maffulli N. Muscles, Ligaments and Tendons Journal. Basic principles and recommendations in clinical and field science research. MLTJ. 2013;4:250-252.

19. Joshi SK, Kim HT, Feeley BT, Liu X. Differential ubiquitinproteasome and autophagy signaling following rotator cuff tears and suprascapular nerve injury. J Orthop Res. 2014;32(1): 138-144.

20. Frey E, Regenfelder F, Sussmann P, et al. Adipogenic and myogenic gene expression in rotator cuff muscle of the sheep after tendon tear. J Orthop Res. 2009;27(4):504-509.

21. Fajas L, Schoonjans K, Gelman L, et al. Regulation of peroxisome proliferator-activated receptor gamma expression by adipocyte differentiation and determination factor $1 /$ sterol regulatory element binding protein 1: implications for adipocyte differentiation and metabolism. Mol Cell Biol. 1999;19(8):54955503.

22. Rosen ED, Walkey CJ, Puigserver P, Speigelman BM. Transcriptional regulation of adipogenesis. Genes Dev. 2000; 14(11):1293-1307.

23. Itoigawa Y, Kishimoto KN, Sano H, Kaneko K, Itoi E. Molecular mechanism of fatty degeneration in rotator cuff muscle with tendon rupture. J Orthop Res. 2011;29(6):861-866.

24. Bodine SC, Stitt TN, Gonzalez M, et al. Akt/mTOR pathway is crucial regulator of skeletal muscle hypertrophy and can prevent muscle atrophy in vivo. Nat Cell Biol. 2001; 3(11):10141019.

25. Premdas J, Tang JB, Warner JP, Murray MM, Spector M. The presence of smooth muscle actin in fibroblasts in the torn human rotator cuff. J Orthop Res. 2006;19(2):221-228.

26. Liu X, Joshi SK, Ravishankar B, Laron D, Kim HT, Feeley BT. Upregulation of transforming growth factor B signaling in a rat model of rotator cuff tears. J Shoulder Elbow Surg. 2014; (11):1709-1716.

27. Rafaello A, Milan G, Masiero E, et al. Jun B transcription factor maintain skeletal muscle mass and promotes hypertrophy. J Cell Biol. 2010;191(1):101-113.

28. Lo IK, Marchuk LL, Hollinshead R, Hart DA, Frank CB. Matrix metalloproteinase and tissue inhibitor of matrix metalloproteinase mRNA levels are specifically altered in torn rotator cuff tendons. Am J Sports Med. 2004;32(5):1223-1229.

29. Zhang Q, Joshi SK, Lovett DH, et al. Matrix metalloproteinase2 plays a critical role I overload induced skeletal muscle hypertrophy. Muscles, Ligaments and Tendons Journal. 2014;4 (3):362-370.
30. Davis ME, Stafford PL, Jergenson MJ, Bedi A, Mendias CL. Muscle fibers are injured at the time of acute and chronic rotator cuff repair. Clin Orthop Relate Res. 2015;473(1):226-232.

31. Bodine SC, Stitt TN, Gonzalez M, et al. Akt/mTOR pathway is crucial regulator of skeletal muscle hypertrophy and can prevent muscle atrophy in vivo. Nat Cell Biol. 2001;3(11):1014-1019.

32. Powers T. Cell growth control: mTOR takes on fat. Mol Cell. 2008;31(6):775-776.

33. Li HY, Zhang QG, Chen JW, Chen SQ, Chen SY. The fibrotic role of phosphatidylinositol-3-kinase/Akt pathway in injured skeletal muscle after acute contusion. Int J Sports Med. 2013;34(9):789-794.

34. Burks TN, Andres-Mateos E, Marx R, et al. Losartan restores skeletal muscle remodeling and protects against disuse atrophy in sacropenia. Sci Transl Med. 2011;3(82):82ra37.

35. Deniz G, Kose O, Tugay A, Gutler F, Turan A. Fatty degeneration and atrophy of rotator cuff muscles after arthroscopic repair: does it improve, halt, or deteriorate? Arch Orthop Trauma Surg. 2014;134(7):985-990.

36. Di Schino M, Augereau B, Nich C. Does open repair of anterosuperior rotator cuff tear prevent muscular atrophy and fatty infiltration? Clin Orthop Relat Res. 2012;470(10):2776-2784.

37. Yamaguchi H, Suenaga N, Oizum N, Hosokawa Y, Kanaya F. Will preoperative atrophy and fatty degeneration of the shoulder muscles improve after rotator cuff repair in patients with massive rotator cuff tears? Adv Orthop. 2012.

38. Chung SW, Kim SH, Tae SK, Yoon JP, Choi Ja, Oh JH. Is the supraspinatus muscle atrophy truly irreversible after surgical repair of rotator cuff tears? Clin Orthop Surg. 2013;5(1):55-65.

39. Jo C, Shin JS. Changes in appearance of fatty infiltration and muscle atrophy of rotator cuff muscles on magnetic resonance imaging after rotator cuff repair: establishing new time-zero traits. Arthroscopy 4. 2013;29(3):449-358.

40. Tong JF, Yan X, Zhu MJ, Du M. AMP-activated protein kinase enhances the expression of muscle-specific ubiquityn ligases despite its activation of IGF-1/Akt signaling in C2C12 myotubes. J Cell Biochem. 2009;108(2):458-468.

41. Osti L, Buda M, Del Buono A. Fatty infiltration of the shoulder: diagnosis and reversibility. Muscles, Ligaments and Tendons Journal. 2013;3(4):351-354.

42. Goutallier D, Postel JM, Bernageau J, Lavau L, Voisin MC. Fatty muscle degeneration in cuff ruptures. Pre- and postoperative evaluations by CT scan. Clin Orthop Relat Res. 1994; 304:78-83.

43. Choo A, McCarthy M, Pichika R, et al. Muscle gene expression patterns in human rotator cuff pathology. J Bone Joint Surg Am. 2014;96(18):1558-1576.

44. Lo IK, Burkhart SS. Arthroscopic repair of massive, contracted, immobile rotator cuff tears using single and double interval slides: technique and preliminary results. Arthroscopy. 2004; 20(1):22-33.

45. Tauro JC. Arthroscopic "interval slide" in the repair of large rotator cuff tears. Arthroscopy. 1999;15:527-530.

46. Kim SJ, Kim SH, Lee SK, Seo JW, Chun YM. Arthroscopic repair of massive contracted rotator cuff tears: aggressive release with anterior and posterior interval slides do not improve cuff healing and integrity. J Bone Joint Surg Am. 2013;95(16): 1482-1488.

47. Van Kampen C, Arnoczky S, Parks P, et al. Tissue-engineered augmentation of a rotator cuff tendon using a reconstituted collagen scaffold: a histological evaluation in sheep. Muscles, Ligaments and Tendons Journal. 2013;3(3):229-235. 